\title{
Enhanced Visualization of Diffusion Tensor Data for Neurosurgery
}

\author{
Frank Enders ${ }^{1,2}$, Dorit Merhof ${ }^{1,2}$, Peter Hastreiter ${ }^{1,2}$, \\ Marc Stamminger ${ }^{2}$ und Christopher Nimsky ${ }^{1,3}$ \\ ${ }^{1}$ Neurocenter, Dept. of Neurosurgery, University of Erlangen-Nuremberg, Germany \\ ${ }^{2}$ Computer Graphics Group, University of Erlangen-Nuremberg, Germany \\ ${ }^{3}$ Dept. of Neurosurgery, University of Erlangen-Nuremberg, Germany
}

\begin{abstract}
Diffusion tensor imaging (DTI) is a recent magnetic resonance (MR) technique that provides comprehensive information about water diffusivity. Since diffusivity correlates with tissue structure at the microscopic level DTI reveals fibrous structures such as white matter (WM) pathways in the field of neurosurgery. WM tracts are thereby of major interest and the ability to distinguish between different WM patterns is subject of growing research. Different approaches for examining the data such as streamline tracking or direct volume rendering have been developed. In this work an integrated method for visualizing this kind of data is presented utilizing glyph-based and streamline visualization in combination with anatomical MR data to obtain a maximum of information.
\end{abstract}

\section{Introduction}

With the introduction of DTI [1,2] a new imaging method providing higher dimensional data is available for medical diagnosis and for inspection during intervention. Particularly neurosurgery benefits from this MR sequence since DTI provides a deeper insight into the structure of the brain in vivo. This is achieved by exploiting the fact that diffusion of water is affected by surrounding tissue. Notably, WM tracts force water to linear diffusion which results in a distinctive anisotropic diffusion characteristic. Against the background of neurosurgery, it is of certain value to have information about the location of WM tracts available for surgical planning which requires a comprehensive visualization strategy $[3,4]$. Due to the huge amount of information contained in the tensor data the software tools must provide utilities for the extraction of the required information with respect to the medical problem.

\section{Material}

For all data acquisition a Siemens MR Magnetom Sonata Maestro Class 1.5 Tesla scanner equipped with a gradient system with a field strength of up to 40 
$\mathrm{mT} / \mathrm{m}$ (effective $69 \mathrm{mT} / \mathrm{m}$ ) and a slew rate of up to $200 \mathrm{~T} / \mathrm{m} / \mathrm{s}$ (effective 346 $\mathrm{T} / \mathrm{m} / \mathrm{s}$ ) was used. With regard to the integrated visualization of DTI data and $\mathrm{MR}_{T 1}$ data the two sequences were gathered back-to-back.

Parameters for DTI were TR $=9200, T E=86 \mathrm{~ms}, \mathrm{~b}_{\text {high }}=1000 \mathrm{~s} / \mathrm{mm}^{2}$, $\mathrm{b}_{\text {low }}=0 \mathrm{~s} / \mathrm{mm}^{2}$, field of view $240 \mathrm{~mm}$, voxel size $1.875 \times 1.875 \times 1.9 \mathrm{~mm}^{3}$, $1502 \mathrm{~Hz} / \mathrm{Px}$ bandwidth, acquisition matrix $128 \times 128$, 60 slices with no intersection gap. The diffusion-encoding gradients for the six diffusion weighted images were directed along the following axes: $( \pm 1,1,0),( \pm 1,0,1)$ and $(1, \pm 1,0)$.

The conventional $\mathrm{MR}_{T 1}$ was acquired with the following parameters:

$\mathrm{TR}=2020 \mathrm{~ms}, \mathrm{TE}=4.38 \mathrm{~ms}$, field of view $250 \mathrm{~mm}$, voxel size $0.488281 \times$ $0.488281 \times 1.0 \mathrm{~mm}^{3}$, acquisition matrix $256 \times 256,160$ slices.

\section{Methods}

The approach is based on the incorporation of three different visualization techniques: Beside direct volume rendering of $\mathrm{MR}_{T 1}$ data that provides anatomical information, fiber and glyph representations of the DTI data are incorporated into the rendering as shown in figure 1 . While direct volume rendering of a $3 \mathrm{D}$ MR dataset is directly possible, DTI datasets require some precomputation to transform the acquired data into data expedient for visualization and simulation.

Therefore, in a first step, a diffusion tensor was computed for each voxel by solving the Stejskal-Tanner equations system [5]. Filter operations were then applied to the tensor field performing 3D gauss filtering for each tensor component.

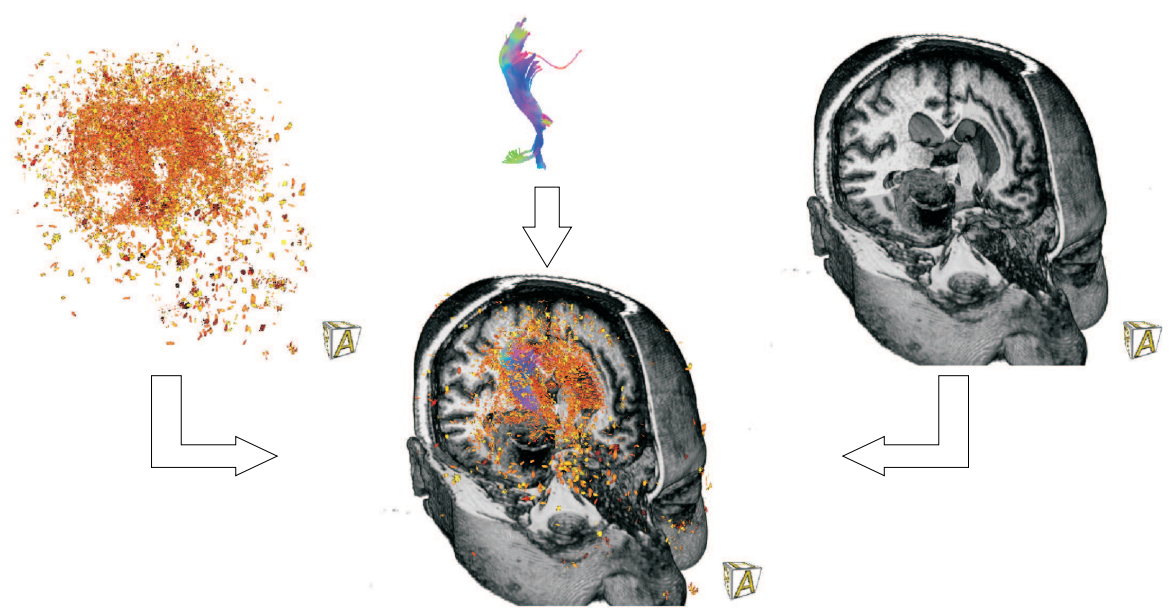

Fig. 1. Fusion of the three different visualization methods glyphs (left), fibers (middle) and direct volume rendering (right) into an integrated rendering (bottom). 
These operations result in a 3D second order tensor field that serves as basis for further processing.

In case of visualizing fibers, streamline tracking had to be performed [6] first. In general, fiber tracking algorithms are based on streamline methods which work on vector fields. The tensor information thus had to be reduced to a vector which was achieved by deriving the eigensystem of a tensor and focusing on the major eigenvector. However, for reasons of accuracy, the tracking algorithm did not evaluate the original tensors directly but used trilinear tensor interpolation before integration using fourth order Runge-Kutta. Fractional anisotropy (FA) was used as tracking threshold to ensure that fibers are only propagated in areas with anisotropic diffusion characteristic.

In case of user-defined regions of interest (ROIs) only fibers traversing those ROIs were displayed. Otherwise, fibers were visualized within the whole brain comprising all WM. Since the resulting structures are rather complex, the display of specific tract systems using ROIs was preferred.

Afterwards, rendering of the fibers was achieved using the OpenGL API. Each tract was thereby stored in a vertex array and was drawn as a set of lines. Color encoding followed the standard strategy using the major eigenvector components as RGB values.

Similar to fiber tracking, glyph-based visualizations require eigensystems instead of direct tensor information. Though, in case of glyphs an eigensystem evaluation per voxel was enough. Interpolation would only be necessary for super-sampling which was not yet applied. Considering the nature of tensors the glyphs are superior to fiber representations since they incorporate the whole tensor information and are not restricted to the major eigenvector. To preserve this advantage the shape of the glyph is crucial. An appropriate shape for representing second order tensors using glyphs is an ellipsoid. Since the polygon model of an ellipsoid would be very cost-intensive for rendering the presented approach uses hardware accelerated ellipsoids as glyphs [7]. They were entirely calculated on the graphics processing unit (GPU) using vertex and fragment shaders to obtain interactive rendering. To avoid overloading of the resulting images with glyphs, again a threshold based on FA was applied. In some cases it was also advantageous to display glyphs only in a single slice (see figure 2). This was of particular interest for the investigation of border areas of tumors.

Finally, volume rendering of anatomical data was included. Since fibers and glyphs were opaque objects they were rendered in advance with enabled depth buffer. Applying direct volume rendering subsequently leads to a correct result.

For enhanced volume rendering, 2D transfer functions were applied leading to an emphasized representation. In addition to that, culling within the volume allowed uncovering hidden structures such as the pyramidal tract or the corpus callosum. To achieve interactive manipulation of an arbitrary clipping geometry an additional 3D texture was generated that was used within a fragment program defining the influence on each voxel. 

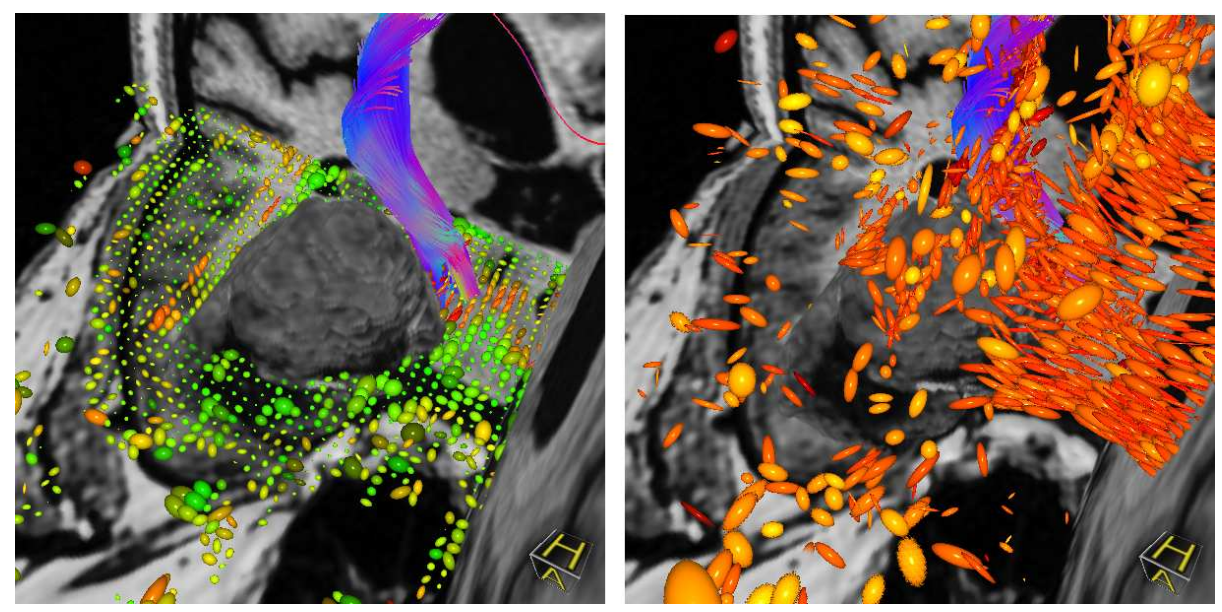

Fig. 2. Comparison of a single slice glyph representation (left) and a $3 \mathrm{D}$ representation (right). FA threshold is set to 0 for single slice display since occlusion is marginal. For $3 \mathrm{D}$ alignment the threshold is set to 185 . FA was mapped between 0 and 255 .

\section{Results}

The approach has been applied to 15 brain tumor cases. In each patient the spatial relation of tumor and pyramidal tract was examined by investigating fibers and glyphs. The ROIs for fiber tracking were manually defined by an expert identifying the internal capsule and the motor cortex. The fusion of tracking results and anatomical data already provided a good spatial impression about white matter tracts and tumor.

Furthermore, the visualization was enhanced by a glyph based rendering of the tensor data showing more comprehensive information at the tumor border for selected slices. Integrating fibers and glyphs into standard $\mathrm{MR}_{T 1}$ data thus provided an improved representation of the information contained within the data and allowed evaluating the reliability of the visualization.

However, it must be mentioned that DTI data shows distortion artifacts in certain areas such as the frontal lobe and the brain stem which are caused by the echo planar imaging sequence used for DTI acquisition. First steps towards non-linear distortion correction were applied. Nevertheless, results in those areas must be treated with caution.

In the context of practical use, a major focus lay on the interactivity of the rendering. This is a crucial aspect since the acceptance of the tool is directly related to the usability and interactive handling. To provide the physician interactively with the maximum of information available, hardware accelerated 3D graphics was applied. 


\section{Conclusion}

We presented a method for interactive display of DTI data. The qualities of fiber tracts which are easy to interpret and glyphs which provide a more comprehensive representation of the data were combined in the presented integrated approach. To further support the investigation of the data, anatomical information was integrated by additionally visualizing $\mathrm{T} 1$ and $\mathrm{T} 2$ weighted MR data using direct volume rendering. Combining all presented visualization techniques leads to a comprehensive display providing an enhanced visualization of the relationship between tumor border and major WM tracts for preoperative planning as well as intra-operative inspection.

\section{Acknowledgments}

This work was supported by the Deutsche Forschungsgemeinschaft in the context of SFB 603, Project C9 and the Graduate Research Center "3D Image Analysis and Synthesis".

\section{References}

1. Basser, P., Mattiello, J., Bihan, D.L.: Estimation of the effective self-diffusion tensor from the nmr spin echo. Journal of Magnetic Resonance B (1994) 247-254

2. Pierpaoli, C., Jezzard, P., Basser, P., Barnett, A., Chiro, G.D.: Diffusion tensor MR imaging of the human brain. Radiology 201 (1996) 637-648

3. Westin, C.F., Maier, S.E., et. al.: Image processing for diffusion tensor magnetic resonance imaging. In: Medical Image Computing and Computer-Assisted Intervention. Lecture Notes in Computer Science (1999) 441-452

4. Kindlmann, G.: Superquadric tensor glyphs. In: Proceedings of Symposium on Visualization 2004. (2004)

5. Stejskal, E., Tanner, J.: Spin diffusion measurements: spin echoes in the presence of a time-dependent field gradient. Journal of Chemical Physics 42 (1965) 288-292

6. Zhukov, L., Barr, A.: Oriented tensor reconstruction: tracing neural pathways from diffusion tensor mri. In: Proceedings of the conference on Visualization '02, IEEE Computer Society (2002) 387-394

7. Klein, T., Ertl, T.: Illustrating Magnetic Field Lines using a Discrete Particle Model. In: Workshop on Vision, Modelling, and Visualization VMV '04. (2004) 